\title{
Experimental Searches on Sterile Neutrinos
}

\section{Michael Wurm*}

Institute of Physics and Excellence Cluster PRISMA, Johannes-Gutenberg University Mainz

E-mail: michael.wurmeuni-mainz.de

While neutrino oscillations among the three active neutrino flavors are by now well established, several anomalies observed at relatively short baselines in oscillation experiments hint towards the existence of one or more additional sterile neutrino flavors. As a consequence, several experiments are in preparation that are dedicated to test the sterile neutrino hypothesis.

The present contribution gives an overview of the on-going experimental efforts, highlighting the two projects that are foreseen to start in 2015: SOX deploying a radioactive neutrino source at short distance from the BOREXINO detector at LNGS, and STEREO searching for sterile neutrino oscillations at short distance from the ILL reactor core.

XIIth International Conference on Heavy Quarks \& Leptons 2014,

25-29 August 2014

Schloss Waldthausen, Mainz, Germany

${ }^{*}$ Speaker. 
Since the discovery of neutrino oscillations around the year 2000, the existence of oscillations between the three active neutrino flavors as well as the basic oscillation parameters (mixing angles $\theta_{i j}$ and mass squared differences $\Delta m_{j i}^{2}$ with $i, j=1,2,3$ ) have been well established. However, there are in fact several experimental hints that there might be oscillation phenomena reaching beyond the standard three-flavor scenario. Due to the LEP bound on the number of active ${ }^{1}$ light neutrinos, any new neutrino flavor added would necessarily be sterile. Therefore, a direct detection of these neutrinos via Standard Model processes is basically excluded. However, additional sterile neutrino $\left(v_{s}\right)$ states may become visible in oscillation experiments via their mixing with the active flavors,

$$
\left(\begin{array}{c}
v_{e} \\
v_{\mu} \\
v_{\tau} \\
v_{s} \\
\vdots
\end{array}\right)=\left(\begin{array}{ccccc}
U_{e 1} & U_{e 2} & U_{e 3} & U_{e 4} & \ldots \\
U_{\mu 1} & U_{\mu 2} & U_{\mu 3} & U_{\mu 4} & \ldots \\
U_{\tau 1} & U_{\tau 2} & U_{\tau 3} & U_{\tau 4} & \ldots \\
U_{s 1} & U_{s 2} & U_{s 3} & U_{s 4} & \ldots \\
\vdots & \vdots & \vdots & \vdots & \ddots
\end{array}\right)\left(\begin{array}{c}
v_{1} \\
v_{2} \\
v_{3} \\
v_{4} \\
\vdots
\end{array}\right),
$$

where the additional mixing coefficients in the expanded PMNS matrix are denoted as $U_{s i}$. In addition, one or several new mass eigenstates $v_{4}$ etc. are introduced, corresponding to the number of additional sterile neutrino states $n$. Such models are commonly denoted as $(3+n)$ scenarios.

In the minimal $(3+1)$ scenario, the existence of the additional sterile neutrino can be deduced from two kinds of oscillation phenomena: In disappearance experiments, oscillations from active to sterile neutrinos (e.g. $v_{e} \rightarrow v_{s}$ ) will result in a deficit in the observed neutrino rates. A useful expression for the oscillation probability as a function of baseline $L$ and neutrino energy $E$ is

$$
P\left(v_{e} \rightarrow v_{e}\right)=1-P\left(v_{e} \rightarrow v_{s}\right)=1-\sin ^{2}\left(2 \theta_{e e}\right) \sin ^{2}\left(\frac{\Delta m_{41}^{2} L}{4 E}\right),
$$

with $\theta_{e e}$ the effective mixing angle. This approximation holds as long as the value of $\Delta m_{41}^{2} \gg$ $\Delta m_{31}^{2}$ and therefore the oscillation length $\ell_{41} \ll \ell_{31}$ (as implied by the current anomalies, sec. 1). Alternatively, the appearance of additional oscillations between the active flavors can be observed at the novel oscillation baseline $\ell_{41}=4 \pi \hbar c \cdot E / \Delta m_{41}^{2}, \ell_{42}$ etc., e.g. denoted by

$$
P\left(v_{\mu} \rightarrow v_{e}\right)=\sin ^{2}\left(2 \theta_{e \mu}\right) \sin ^{2}\left(\frac{\Delta m_{41}^{2} L}{4 E}\right) .
$$

Independently of oscillations, the existence of a fourth mass eigenstate and the size of its admixture to the $v_{e}$ flavor state can also be approached by experiments scrutinizing the endpoint region of $\beta$ decay spectra. Compared to the standard three-flavor scenario, the new mass eigenstate will cause a distortion of the decay spectrum.

In the following, the case for a possible fourth sterile neutrino state will be made based on the anomalies in present oscillation data (sec. 1). New experimental ideas for an unambiguous verification or rejection of this hypothesis will be presented in secs. 2-4, highlighting in particular the short-baseline reactor and source experiments STEREO and SOX. Finally, the alternative approach of investigating the tritium decay spectrum in KATRIN will be introduced in sec. 5 before concluding in sec. 6 .

\footnotetext{
${ }^{1}$ i.e. coupling to weak interaction via $Z^{0}, W^{ \pm}$.
} 


\section{Motivation for sterile neutrino searches}

The possible evidence for the existence of sterile neutrinos results from the data of several oscillation experiments that are in tension with the standard three-flavor picture:

Gallium anomaly. The anomaly arises from the calibration tuns performed in the solar radiochemical experiments Gallex/GNO and SAGE. In order to validate the cross-sections and extraction efficiencies that are at the heart of the translation of observed event rates to solar neutrino fluxes, both collaborations performed calibration measurements with strong radioactive neutrino sources. The sources used for this purpose were based on the isotopes ${ }^{51} \mathrm{Cr}$ and ${ }^{37} \mathrm{Ar}$ and provided activities of about $1 \mathrm{MCi}$. Both radioisotopes undergo electron capture (EC), emitting a mono-energetic $v_{e}$ of about $0.8 \mathrm{MeV}$. In a total of four runs, the sources were placed inside the respective experimental setups and therefore at very short distance from the neutrino target. The resulting rates have been evaluated to be on average only $0.86 \pm 0.05$ of the expectation (deviating at $2.8 \sigma$ from unity) [1].

Reactor anomaly. Nuclear reactors have been identified early as powerful $\bar{v}_{e}$ sources and have been used in a multitude of oscillation experiments. When early experiments close to the reactor cores reported $\bar{v}_{e}$ rates close to the expectation values, detectors moved further and further away from the cores and finally discovered oscillations at distances of $\sim 1 \mathrm{~km}\left(\theta_{13}\right)$ and $\sim 120 \mathrm{~km}\left(\theta_{12}\right)$. However, a re-evaluation of the neutrino spectra and fluxes edited by reactors has been performed based on the state-of-the-art of nuclear data and theory in 2011. The study implies that the formerly used prediction for the $\bar{v}_{e}$ event rates was low by $4.5 \%[2,3]$. Based on the new results, there arises a deficit in the rates detected by experiments at distances shorter than $\sim 100 \mathrm{~m}$ from the respective reactors. The $v_{s}$ white paper [4] places the significance of the rate deficit at the $3 \sigma$ level.

Accelerator anomalies. There is a long-standing anomaly in the data of the short-baseline oscillation experiment LSND at FNAL. LSND searched for oscillation of type $\bar{v}_{\mu} \rightarrow \bar{v}_{e}$ at a low energy $\bar{v}_{\mu}$ source based on stopped- $\pi^{+}$-decay. After observing an unexplainable excess in $\bar{v}_{e}$-like events $(3.8 \sigma)$ [5], the MiniBooNE experiment was devised to cross-check the result at higher energies and longer baselines (providing the same distance-over-energy $L / E$ ratio) and testing both neutrino and antineutrino mode. As LSND, MiniBooNE observed anormal appearance signals for both beam polarities [6].

While the significances of the individual anomalies are relatively weak, a more compelling picture is arising when all of the experimental results are regarded in combination. They can be consistently interpreted as oscillations via a novel mass squared difference $\Delta m_{41}^{2} \approx 1.5 \mathrm{eV}^{2}$. The accompanying light neutrino flavor state must be sterile. In this picture, gallium and reactor anomalies can be understood as $v_{e} \rightarrow v_{s}$ disappearance oscillations at an amplitude of $\sin ^{2} 2 \theta_{e e} \approx 0.15$ (eq. 2). The LSND and MiniBooNE $v_{e}$-like excess would correspond to appearance oscillations $\bar{v}_{\mu} \rightarrow \bar{v}_{e}$ mediated by the new mass eigenstate (eq. 3). Therefore, all anomalies could be explained by a $(3+1)$ scenario with the known three active plus one additional sterile neutrino. A review of the evidence in favor of sterile neutrinos can be found in [4], while a global analysis of all available oscillation data has been performed in [7]. Figure 1 shows the allowed oscillation parameter space for oscillations between $v_{e}, v_{\mu}$ and $v_{s}$. 

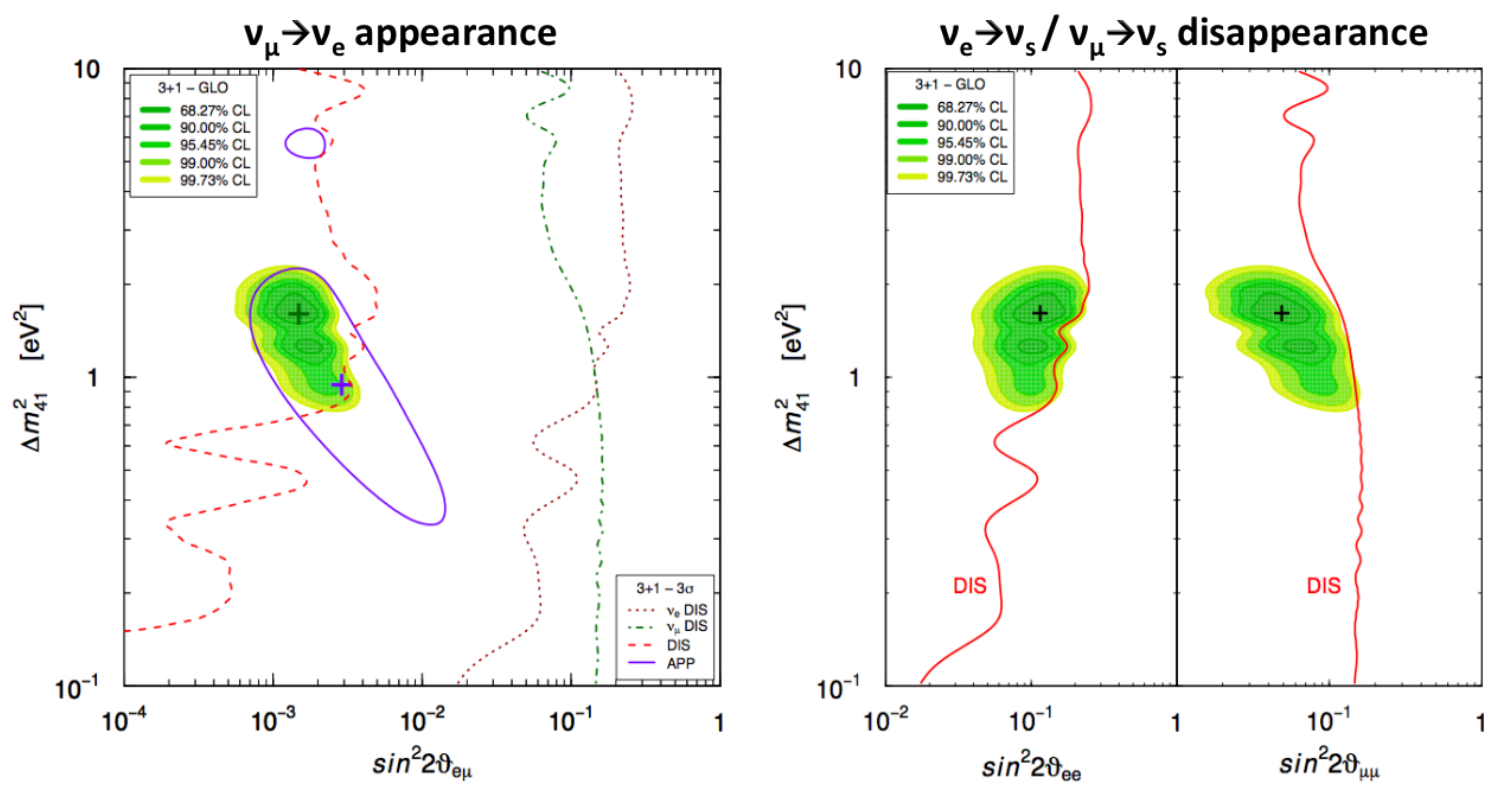

Figure 1: The allowed parameter space for sterile neutrino oscillation as derived by a global analysis of oscillation data in [7].

\section{Reactor experiments}

The reactor anomaly describes a distance-independent suppression of the observed event rates at baselines shorter than $100 \mathrm{~m} \mathrm{[3].} \mathrm{However,} \mathrm{none} \mathrm{of} \mathrm{the} \mathrm{experiments} \mathrm{relevant} \mathrm{for} \mathrm{the} \mathrm{anomaly}$ was close enough to the reactor core to detect a variation of the neutrino rate with either energy or distance (eq. 2) consistent with the currently preferred $v_{s}$ oscillation parameters ${ }^{2}$ (sec. 1). To obtain a definite confirmation of oscillations $\bar{v}_{e} \rightarrow \bar{v}_{s}$ as the origin of the anomaly, future experiments will be positioned very close to the reactor core, at baselines of the order of $10 \mathrm{~m}$ or less. Moreover, the detectors have to be sensitive to either the neutrino energy or the vertex of the neutrino interaction in order to resolve the emerging oscillation pattern in the $\bar{v}_{e}$ survival probability. The primary challenge is the very high gamma and neutron background level expected at such short distance from a reactor core.

Triggered by the discovery of the reactor anomaly in 2011, a large number of sterile neutrino projects at reactors started all over the world. Ordered by their hosting countries, the nonexhaustive list [9] includes the SOLID experiment at BR2 reactor (Belgium), the French NUCIFER at Osiris reactor (Saclay) and STEREO at ILL Grenoble, three Russian experiments (DANSS at KNPP Udomlya, NEUTRINO4 at SM-3 Dimitrovgrad, andPOSEIDON at PIK Gatchina) as well as PROSPECT at HFIR reactor (ORNL) in the US.

Of these proposed projects, the STEREO experiments at the ILL research reactor is one of the most advanced [9]. Due to the compact reactor core geometry, the neutrino emission region is limited to less than $1 \mathrm{~m}$ diameter, mitigating the smear-out of the oscillation signature and allowing for a detector very close to the source (distance of 9 to $11 \mathrm{~m}$ ). The chosen target medium is a

\footnotetext{
${ }^{2}$ With the noteworthy exception of the ILL experiment that was positioned about $9 \mathrm{~m}$ from the Grenoble reactor core and observed a signal compatible with short-baseline oscillations [8].
} 
gadolinium-doped liquid scintillator in which $\bar{v}_{e}$ are detected by the inverse beta decay on free protons, $\bar{v}_{e}+p \rightarrow e^{+}+n$. While the prompt positron signals provides precise information on the neutrino energy, the delayed capture of neutrons on gadolinium provides a delayed signal that can be used for an efficient reduction of single-event background. Moreover, spatial resolution is assured by segmenting the detector in six optically separated sub-modules. The target cells are surrounded by passive shielding and active veto layers, the latter allowing to study and reject events induced by external backgrounds.

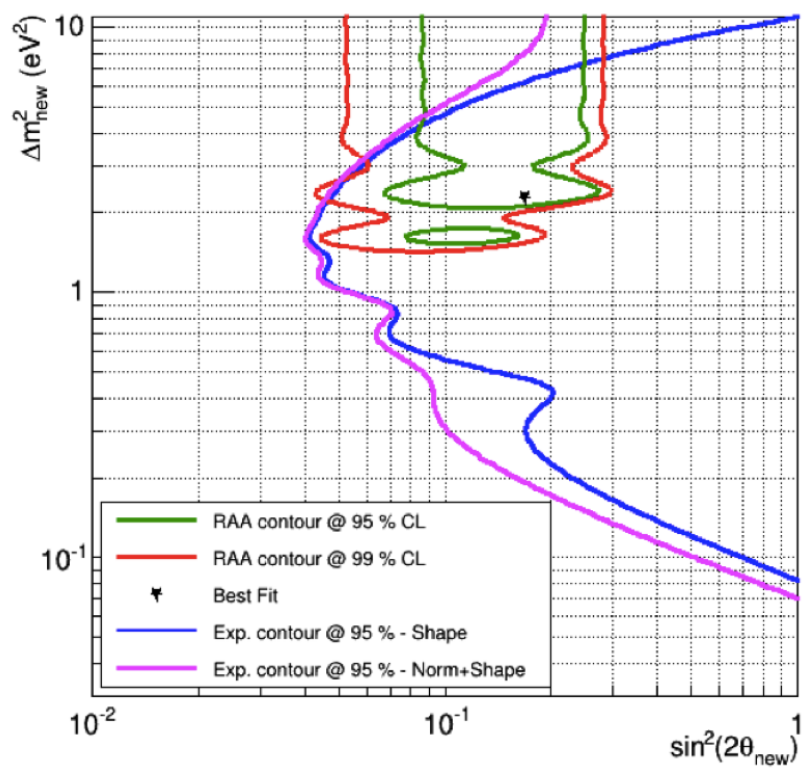

Figure 2: Sensitivity of the STEREO reactor experiment:The oscillation parameter space to be excluded by the experiment fully covers the best-fit region of the reactor antineutrino anomaly [9].

The STEREO collaboration projects the start of measurement for 2015. The expected neutrino rate is $\sim 400 \bar{v}_{e}$ events per day at a distance of $10 \mathrm{~m}$ from the reactor core. Figure 2 reproduces the experimental sensitivity that has been calculated based on 300 days detector life time, a $2 \%$ relative error on the energy scale and a signal-to-background ratio of 1.5 [9]. Under these assumptions, the best-fit region of the reactor anomaly will be fully covered.

\section{Radioactive source experiments}

As in the case of the reactor anomaly, the source calibration data of the radiochemical experiments at the base of the gallium anomaly do not provide energy or distance information. However, $\beta$ decay or EC isotopes are considered an attractive neutrino source as they are mobile and can be integrated into the setups of already existing neutrino detectors. Moreover, the background produced by the sources is easier to shield than the intense radiation present in immediate proximity of a reactor core. Finally, these experiments offer the possibility to investigate oscillations both in the neutrino $\left(\beta^{+}\right)$and antineutrino $\left(\beta^{-}\right)$sector.

One of the proposed ideas is to re-check the gallium calibration results based on a segmented detector providing spatial sensitivity. For this, the proposed BEST experiment would re-arrange 
the setup of the SAGE experiment at Baksan (Russia), placing a ${ }^{51} \mathrm{Cr} v_{e}$ source at the center of the detector assembly. However, most of the source experiments have been discussed in the context of the major low-energy neutrino observatories based on the liquid-scintillator technology. These detectors provide both precise energy and spatial information and will allow to test a large fraction of the preferred parameter space. The list of proposed experiments includes running detectors (Daya Bay, KamLAND, Borexino) as well as projects planned for the near or medium future (SNO+, LENS, JUNO, LENA).

Of these proposals, the closest to completion is the SOX experiment in which the neutrino source will be positioned outside but very close to the Borexino detector at the Gran Sasso National Laboratory (LNGS) [10]. A suitable pit located below the spherical detection volume at $8.25 \mathrm{~m}$ distance from the detector center offers the possibility to host either a mono-energetic $v_{e^{-}}$ source based on ${ }^{51} \mathrm{Cr}\left(E_{v} \approx 750 \mathrm{keV}, A=10 \mathrm{MCi}\right.$, produced by $n$-irradiation of $\left.{ }^{50} \mathrm{Cr}\right)$ or a $\bar{v}_{e}$-source relying on the short decay chain of the radioisotopes ${ }^{144} \mathrm{Ce}-{ }^{144} \operatorname{Pr}\left(E_{V} \leq 3.0 \mathrm{MeV}, A=100 \mathrm{kCi}\right.$, extracted from spent nuclear fuel). Preparations for the latter option are by now far progressed and a deployment of the source is foreseen for end of 2015.

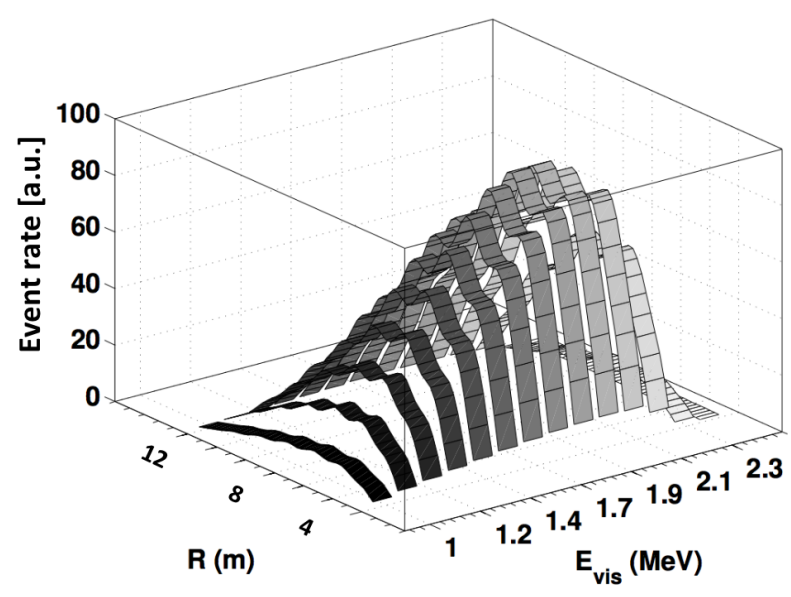

Figure 3: The energy and distance-dependent oscillation pattern in SOX with a ${ }^{144} \mathrm{Ce} \bar{v}_{e}$-source.

The signature for sterile neutrinos in SOX is the emergence of a disappearance oscillation pattern in the position and energy of the $\sim 10^{4} v_{e} / \bar{v}_{e}$ events reconstructed in the detection volume (figure 3). As in the case of reactor antineutrinos, the relevant detection reaction for the Ce source is the inverse beta decay, limiting the range of detectable neutrino energies to $1.8-3.0 \mathrm{MeV}$. For the preferred value of $\Delta m_{41}^{2} \approx 1.5 \mathrm{eV}^{2}$, the corresponding oscillation lengths range from $3 \mathrm{~m}$ to $5 \mathrm{~m}$ and are therefore well contained within the target volume of $8.5 \mathrm{~m}$ diameter. The delayed neutron capture signal on hydrogen provides a powerful tagging signature and reduces the background from single-event radioactivity to a negligible level. The background from antineutrino(-like) signals of reactor and geoneutrinos as well as cosmogenic backgrounds will be virtually negligible.

Figure 4 displays the expected sensitivity of SOX both for the ${ }^{144} \mathrm{Ce} \bar{v}_{e}$ and the ${ }^{51} \mathrm{Cr} v_{e}$ sources [11]. Sensitivity is greatest around the preferred range of $\Delta m_{41}^{2}$ where the oscillation lengths are of the order of magnitude of the detector diameter. Sensitivity vanishes for lower values of $\Delta m_{41}^{2}$ if $\ell_{41}$ greatly exceeds the detector dimenions, while the sensitivity is also somewhat reduced for high 
values of $\Delta m_{41}^{2}$ where $\ell_{4} 1$ falls below the spatial resolution of BOREXINO ( $\sim 10 \mathrm{~cm}$ at a visible energy of $1 \mathrm{MeV}$ ). The most important systematic uncertainties are related to the normalization of the (non-oscillated) event rate: The definition of the fiducial volume by spatial reconstruction (to be determined in a dedicated calibration campaign) and the decay rate of the Ce source (measured by calorimetry of its thermal power). For the projected uncertainty of $\sim 1 \%$ for both quantities, SOX will be able to exclude the $95 \%$ preferred region of the anomalies at $95 \%$ C.L. (fig. 4).

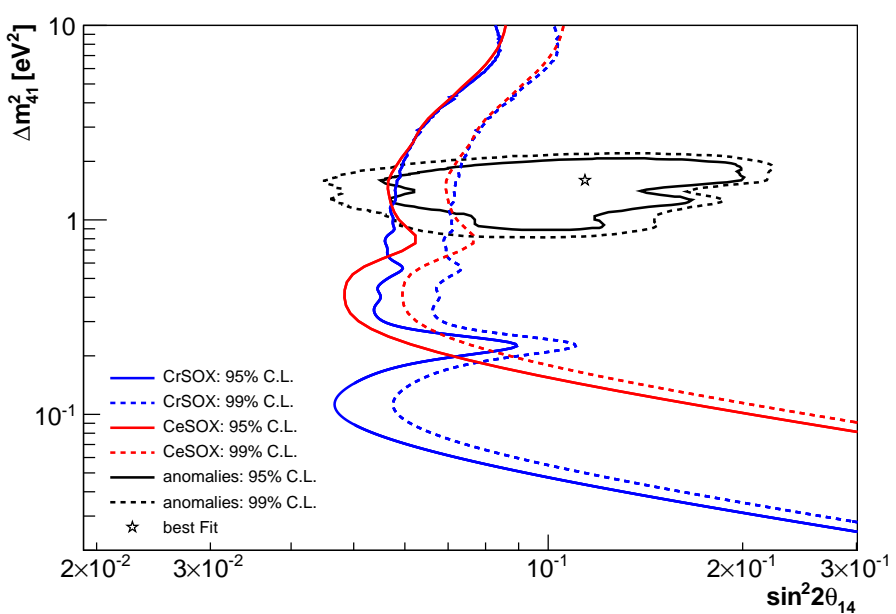

Figure 4: Sensitivity of the SOX source experiment:The oscillation parameter space to be excluded by the experiment covers the best-fit region of the anomalies [11].

\section{Accelerator experiments}

As neutrino beam experiments allow for an accurate adjustment of energy spectra, baselines and detector performance as well as the search for sterile neutrino oscillations in both appearance and disappearance modes, many interesting proposals for precision tests of the $v_{s}$ anomalies are currently discussed.

Compared to LSND and MiniBooNE, new projects aim to improve the knowledge of the initial beam spectrum, the involved neutrino detection cross sections as well as the definition of the energy scale. For these ends, a next-generation experiment will most likely be equipped with a near detector for measuring the un-oscillated neutrino signal. As both LSND and MiniBooNE suffered from relatively high backgrounds in the region of interest for the appearance signal, future experiments will try to reduce backgrounds: In this context, liquid-argon time projection chambers (LAr-TPCs) are currently discussed for both near and far detectors. Finally, event statistics can be improved by both increasing the beam power and enlarging the detection volumes.

Current time scales for the proposed experiments can be expected to be substantially longer than for the reactor and source experiments. While the MicroBooNE LAr-TPC is already running at FNAL and will provide both a test on the feasibility of future detectors and valuable information on neutrino cross-sections [12], statistics will most likely not be sufficient to shed new light on the sterile neutrino anomalies. Far greater sensitivity might be reached in a recurringly discussed 
project with two large LAr-TPCs at FNAL [13]. For this, one or both of the ICARUS T-300 modules would be transported from LNGS to FNAL to serve as a far detector, while the place of the near detector could be taken by the planned LAr1 experiment.

\section{Beta-decay endpoint measurement}

A further and quite complementary approach to search for a fourth $\mathrm{eV}$-scale neutrino state is the investigation of the end-point region of $\beta$ decay spectra. The current best limits on the electron neutrino mass from laboratory experiments stem from experiments studying the shape of the tritium decay endpoint. The up-coming KATRIN experiment is expected to further improve the current limit of $m_{\beta} \leq 2 \mathrm{eV}^{2}$ to the regime of $200 \mathrm{meV}$ [14].

Recent studies indicate that this sensitivity might be sufficient to resolve the the fourth mass state $v_{4}$ by its admixture to the $v_{e}$ flavor state. For the predicted values of $\Delta m_{41}^{2}$ and $\sin ^{2}\left(2 \theta_{e e}\right)$, the corresponding deformation of the $\beta$ decay spectrum close to the endpoint will be right on the verge of the experimental sensitivity. Figure 5 shows the results of an analysis performed in [14] that takes only statistical uncertainties into account. In a $(3+1)$ scenario, the depicted parameters $\Delta m_{s}^{2}$ and $\sin ^{2}\left(2 \theta_{s}\right)$ can be directly associated $\Delta m_{41}^{2}$ resp. $\sin ^{2}\left(2 \theta_{e e}\right)$.

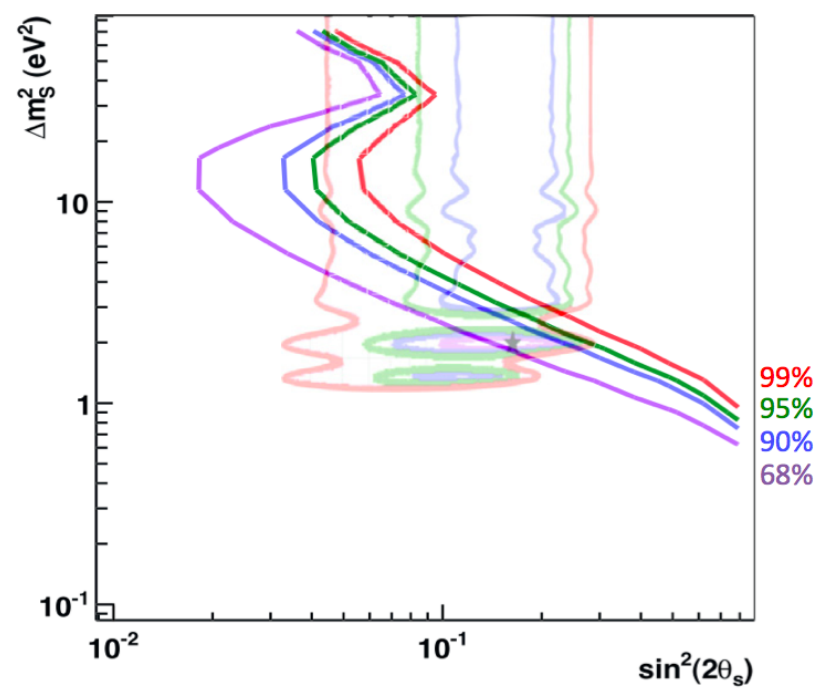

Figure 5: Sensitivity of the KATRIN experiment to an admixture of the mass eigenstate $v_{4}$ to the flavor state $v_{e}$ :The oscillation parameter space to be excluded by the experiment reaches well into the best-fit region of the anomalies [14]. Lines of different colors indicate the confidence levels of the exclusion.

\section{Conclusions}

While the oscillations between the three active neutrino flavors are by now well established, several experiments have provided hints that the active neutrinos might be mixing with at least one further light sterile neutrino flavor. A multitude of experiments has been proposed to test the sterile neutrino hypothesis, either by finding the un-ambiguous evidence of a neutrino oscillation pattern at short baselines or by refuting the currently allowed parameter region. The two projects that are 
by now the most advanced are the STEREO experiment at the ILL reactor in Grenoble and the SOX source experiment at the BOREXINO detector at Gran Sasso. Both experiments are foreseen to start during 2015 and to provide first data the year after. The KATRIN experiment will provide complementary data by searching for a deformation of the tritium $\beta$-decay endpoint region.

\section{References}

[1] C. Giunti and M. Laveder, Phys. Rev. C 83 (2011) 065504 [arXiv:1006.3244].

[2] T. A. Mueller et al., Phys. Rev. C 83 (2011) 054615 [arXiv:1101.2663].

[3] G. Mention et al., Phys. Rev. D 83 (2011) 073006 [arXiv:1101.2755].

[4] K. N. Abazajian et al., arXiv:1204.5379 [hep-ph].

[5] A. Aguilar-Arevalo et al. [LSND Collaboration], Phys. Rev. D 64 (2001) 112007 [hep-ex/0104049].

[6] A. A. Aguilar-Arevalo et al. [MiniBooNE Collaboration], Phys. Rev. Lett. 110 (2013) 161801 [arXiv:1207.4809, arXiv:1303.2588].

[7] C. Giunti, M. Laveder, Y. F. Li, Q. Y. Liu and H. W. Long, Phys. Rev. D 86 (2012) 113014 [arXiv:1210.5715].

[8] H. Kwon et al., Phys. Rev. D 24 (1981) 1097.

[9] D. Lhuillier, PoS EPS -HEP2013 (2013) 522 \& talk at XXVI Int. Conference on Neutrino Physics and Astrophysics, Boston (2014).

[10] G. Bellini et al. [Borexino Collaboration], JHEP 1308 (2013) 038 [arXiv:1304.7721].

[11] B. Caccianiga, talk at XXVI Int. Conference on Neutrino Physics and Astrophysics, Boston (2014).

[12] C. M. Ignarra, FERMILAB-2014-20.

[13] M. Antonello et al., FERMILAB-PROPOSAL-1052.

[14] J. A. Formaggio, J. Barret, PLB 706 (2011) 68 